\section{SPAWAR

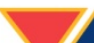

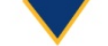 \\ Systems Center}

PACIFIC

TECHNICAL REPORT 2070

December 2014

\title{
Sparse Representation and Dictionary Learning as Feature Extraction in Vessel Imagery
}

Katie Rainey

Ana Ascencio 


\section{SSC Pacific \\ San Diego, California 92152-5001}

\section{ADMINISTRATIVE INFORMATION}

The work described in this report was performed for the Office of Naval Research and the National Geospatial-Intelligence Agency by the Advanced Analysis Systems Branch (Code 56220) and the Atmospheric Propagation Branch (Code 55280), Space and Naval Warfare Systems Center Pacific (SSC Pacific), San Diego, CA.

Released by H. L. Buck, Head Advanced Analysis

Systems Branch
Under authority of

C. A. Wilgenbusch, Head ISR Division

\section{ACKNOWLEDGMENTS}

This work grew from discussions with Alexey Castrodad, Jack Cooper, Shibin Parameswaran, and Josh Harguess. The authors would like to thank them for their ideas and support.

This is a work of the United States Government and therefore is not copyrighted. This work may be copied and disseminated without restriction. 


\section{EXECUTIVE SUMMARY}

\section{OBJECTIVE}

This report describes experiments designed to evaluate the usefulness of a specific algorithm for classifying images of commercial ships by class. This algorithm uses a technique known as sparse coding to represent images for classification. The sparse coding algorithm is compared with another algorithm evaluated in previous publications.

\section{RESULTS}

The sparse coding algorithm is shown to perform approximately as well as the algorithm it is compared with and does not appear to offer any improvement.

\section{RECOMMENDATIONS}

Additional research is required to identify algorithms best suited for the ship classification task. 


\section{CONTENTS}

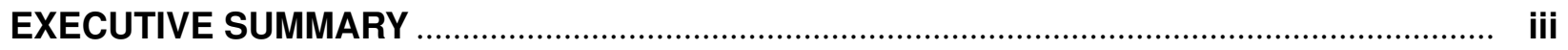

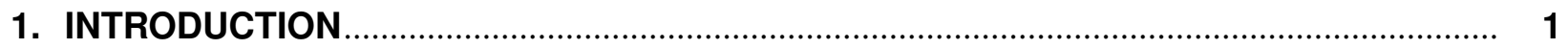

2. DESCRIPTION OF ALGORITHMS …........................................................................... 2

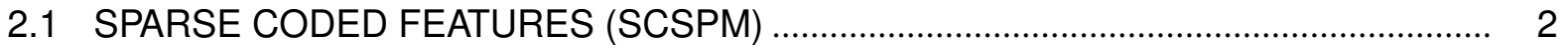

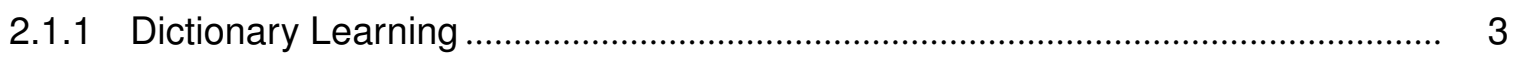

2.1.2 Spatial Pyramid Matching ..................................................................................... 3

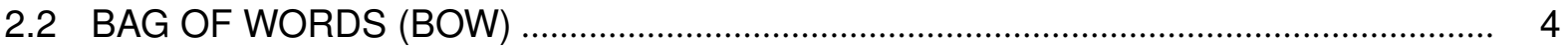

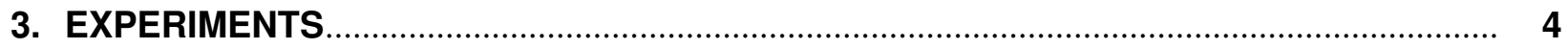

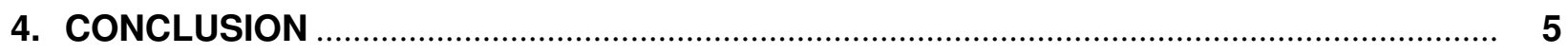

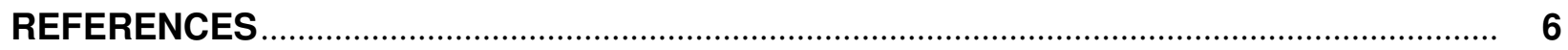

\section{Table}

1. Average classification accuracies on various data sets using either BOW or ScSPM image representations 


\section{INTRODUCTION}

Automated vessel detection and recognition is an important goal for many Navy applications. The automated classification of merchant ships would assist imagery analysts and provide greater maritime domain awareness. This is a challenging problem, in part due to the nature of ship imagery. Ship recognition algorithms must be able to handle variations in resolution and illumination conditions and broadly defined ship categories.

Previous work by researchers at Space and Naval Warfare Systems Center Pacific (SSC Pacific) [5, 12-15] investigated several recognition algorithms and tested them on several data sets of ship images from satellite imagery. Those works considered various methods for image representation and classification, two general steps in the image recognition process. A digital image can be represented as a vector of pixel values, or through a more involved algorithm that attempts to capture semantic value from the pixels. Once an image is represented numerically, that representation can be passed to a classifier which will apply a semantic label to the image. Several algorithms for representation and classification are detailed in [13].

Some of the highest accuracy results in that work were obtained by constructing image representations with the bag of (visual) words (BOW) method [19]. The BOW algorithm first extracts local feature descriptors from an image using the Scale-Invariant Feature Transform (SIFT) [8]. The descriptors are then clustered and pooled with respect to a dictionary of vocabulary features obtained from training imagery. The image is represented as a histogram of its pooled features. One advantage this method has over other representation methods is that the dimensions of its output representation are independent of the dimensions of its input image, which means that two images with different sizes will have the same size representations. This makes the BOW method useful for data sets of images with non-uniform dimensions, such as the ship data described in [14]. Several variations on the BOW method were compared in [13].

Several classification methods were investigated in [13], including Support Vector Machines (SVM), which have been used to much success for many different recognition tasks [16]. An SVM is a type of linear classifier that is designed to maximize the margin of the decision boundary between positive and negative examples, or support vectors. The highest accuracy rates in [13] were achieved with BOW image representations and SVM classifiers.

Another classification method considered in [13], sparse representation-based classification (SRC), classifies an image representation by first expressing it as a sparse linear combination of the columns of a dictionary matrix learned from training images. This method was used by Wright, Yang, Ganesh, Sastry, and Ma [18] to classify images of faces represented by randomly selected pixel values.

Sparse codes have also been used for image representation, rather than for classification. An algorithm referred to as $\mathrm{ScSPM}^{1}$ has been used for image representation by some authors as a replacement for BOW $[6,21]$. In the BOW method, SIFT descriptors are extracted and then quantized with respect to a dictionary. The quantization step means that each descriptor is associated with the one dictionary element to which it is most similar. The ScSPM method replaces quantization with sparse coding; each descriptor is associated with the coefficients of a linear combination of dictionary elements that approximates the descriptor. More information about a descriptor can be retained through sparse coding, since it can be associated with more than one dictionary element.

Any number of SIFT descriptors may be extracted from a given image, so it is necessary to some how pool the quantized descriptors into a single representation of the image. The BOW method pools descriptors into a histogram, but this discards any spatial information from the descriptors. The ScSPM method

\footnotetext{
${ }^{1}$ Similar techniques were used by Yang, Yu, Gong, and Huang [21], who called their method ScSPM to refer to sparse coding and Spatial Pyramid Matching, and by Ji, Theiler, Chartrand, Kenyon, and Brumby [6], who call their method SIFT-based sparse coding.
} 
pools descriptors using a Spatial Pyramid Matching (SPM) algorithm [7] that concatenates histograms from different regions of the image at multiple scales.

In this report, we compare the effectiveness of the ScSPM algorithm for classifying ship imagery with that of BOW. Both algorithms are used to represent ship images, and the representations are fed into an SVM for classification. Our results show similar classification accuracy using both methods, and suggest that some of the perceived advantages of ScSPM may not make a difference with certain data sets. This report is organized as follows: the algorithms considered are detailed in Section 2, the experiments are described in Section 3, and the report is concluded in Section 4.

\section{DESCRIPTION OF ALGORITHMS}

We perform image classification using two different methods for image representation-ScSPM and BOW-each combined with an SVM classifier. This section describes the details of the implementations of the two image representation methods.

Both image representation methods are based on SIFT feature descriptors. Typically, the SIFT algorithm identifies salient keypoints in an image, then computes 128-dimensional descriptors of the region surrounding the keypoints using a histogram of local gradients. These descriptors can be used to match an object in different images, even under changes of scale or illumination. For this work, we use two variations on this algorithm. Dense SIFT computes SIFT descriptors at a dense grid of points, rather than at keypoints. This implementation is faster than traditional SIFT. Dense SIFT extracts features at a single scale, but Bosch, Zisserman, and Muoz [1] proposed a method called pyramid histogram of visual words (PHOW) which extracts dense SIFT features at multiple scales. Both dense SIFT and PHOW are implemented using the VLFeat library [17]. For either method, given an image $\mathbf{X}$, we compute a set of $p$ descriptors, $\psi=\mathbf{y}_{1}, \mathbf{y}_{2}, \cdots \mathbf{y}_{p}$, with $\mathbf{y}_{i} \in \mathbb{R}^{128}$ for each $i=1, \cdots, p$. The value of $p$ varies with each image.

Both representation methods also rely on a dictionary formed from a set of $M$ training images,

$$
\chi_{T}=\left\{X_{1}, \cdots, X_{M}\right\} .
$$

Each training image $X_{j}$ provides $p_{j}$ descriptors, so we obtain a set

$$
\psi_{T}=\left\{\mathbf{y}_{1}^{(1)}, \cdots, \mathbf{y}_{p_{1}}^{(1)}, \cdots, \mathbf{y}_{1}^{(M)}, \cdots, \mathbf{y}_{p_{M}}^{(M)}\right\}
$$

containing all of the descriptors for all of the training images. Methods for constructing a dictionary from these descriptors are described in the sections below.

\subsection{SPARSE CODED FEATURES (SCSPM)}

The aim of sparse coding is to express an input signal $\mathbf{y} \in \mathbb{R}^{n \times 1}$ as a linear combination of the columns a dictionary matrix $\mathbf{D} \in \mathbb{R}^{n \times K}$. The coefficients of this linear combination are stored in the vector $\mathbf{a} \in \mathbb{R}^{K \times 1}$, which can be used to represent the input $\mathbf{y}$. That is, we want to solve the expression

$$
\mathbf{y}=\mathbf{D a}
$$

for a. D should be overcomplete $(K>>n)$ to ensure that there are more than enough columns with which to express any given input $\mathbf{y}$. If $\mathbf{D}$ is overcomplete, then there exist infinitely many solutions to 
Equation (1). We want a to be sparse, with most of its entries zero, which means that only a few of the columns of the dictionary contribute significantly to the representation of $\mathbf{y}$. Therefore we want to find the sparsest possible solution to Equation (1). This requirement can be expressed as

$$
\underset{\mathbf{a} \in \mathbb{R}^{K}}{\operatorname{argmin}} \Omega(\mathbf{a}) \quad \text { such that } \mathbf{y}=\mathbf{D a},
$$

or

$$
\underset{\mathbf{a} \in \mathbb{R}^{K}}{\operatorname{argmin}}\|\mathbf{y}-\mathbf{D a}\|_{2}^{2}+\lambda \Omega(\mathbf{a})
$$

where $\Omega(\cdot)$ is a sparsity-enforcing function and $\lambda$ is a weighting parameter. The immediately obvious choice for $\Omega$ is the so-called $\ell_{0}$ "norm" $\|\cdot\|_{0}$ that counts the number of non-zero entries of a vector, but that makes Problem (3) non-convex and therefore computationally challenging to solve. Another option for $\Omega$ is the $\ell_{1}$ norm $\|\cdot\|_{1}$, a true norm. This option has been shown to yield sparse solutions [3].

\subsubsection{Dictionary Learning}

Many options exist for constructing a dictionary from a set of training images $\chi_{T}$. To begin with, we use the dense SIFT algorithm to compute the set $\psi_{T}$ containing all of the SIFT descriptors from all of the training images, then randomly select $K$ descriptors from this set. The $K$ descriptors can simply be concatenated into a matrix that can serve as a dictionary, but that may not lead to especially sparse solutions. A pre-defined dictionary of basis vectors, such as one constructed with wavelets, may be used instead. Much research has been conducted into how best to learn a dictionary that is specially adapted to training data and that lends itself to sparse representation, and how the dictionary can be computed efficiently [10]. Some dictionary learning algorithms have been designed for a specific task such as classification [11] or image denoising [4]. Several dictionary learning methods are compared in [6] for their use for sparse coding of image features. In the experiments described in this report, we use the SPArse Modeling Software (SPAMS) package $[9,10]$ to solve the $\ell_{1}$-regularized problem

$$
\underset{\mathbf{D} \in \mathbb{R}^{n \times K}}{\operatorname{argmin}} \frac{1}{N_{T}} \sum_{\mathbf{y} \in \psi_{T}} \min _{\mathbf{a} \in \mathbb{R}^{K}} \frac{1}{2}\|\mathbf{y}-\mathbf{D a}\|_{2}^{2}+\lambda\|\mathbf{a}\|_{1},
$$

where $N_{T}$ is the number of signals in the training set $\psi_{T}$, and $K$ is a fixed value indicating the desired size of the dictionary.

We next use our learned dictionary $\mathbf{D}$ to compute the ScSPM representation of an image $\mathbf{X}$. For each $\mathbf{y} \in \psi$, where $\psi$ is the set of dense SIFT feature descriptors extracted from $\mathbf{X}$, we find the sparse code $\mathbf{a}$ with respect to $\mathbf{D}$ by solving Problem (3). We solve the $\ell_{1}$ regularization of (3), with $\Omega(\cdot) \equiv\|\cdot\|$, using the SPAMS package. This leaves us with a set $\mathbf{A}_{X}=\left\{\mathbf{a}_{1}, \cdots, \mathbf{a}_{p}\right\}$ containing the $p$ sparse codes corresponding to the $p$ dense SIFT descriptors from the image $\mathbf{X}$.

\subsubsection{Spatial Pyramid Matching}

Our next step is to pool the $p$ descriptors into a single representation of $\mathbf{X}$, for which we use the Spatial Pyramid Matching (SPM) algorithm proposed by Lazebnik, Schmid, and Ponce [7]. We divide $\mathbf{X}$ into three different partitions, $4 \times 4,2 \times 2$, and $1 \times 1$, for a total of 21 segments. Each segment is home to a subset of descriptors of $\mathbf{X}$ collected in $\psi$; we notate the subset of $\psi$ associated with the $l$ th segment by $\psi_{l}$. We then pool all of the descriptors from a given segment by selecting the maximum value component-wise, giving us the vector $\mathbf{z}_{l}=\left[z_{j}^{(l)}\right] \in \mathbb{R}^{K}$, where for each $j=1, \cdots, K$,

$$
z_{j}^{(l)}=\max _{\mathbf{a} \in \psi_{l}} a_{j}
$$


where $a_{j}$ is the $j$ th component of $\mathbf{a}$.

At this point we have 21 vectors $\mathbf{z}_{1}, \cdots, \mathbf{z}_{21} \in \mathbb{R}^{K}$. By pooling vectors from the segments of several partitions, we are capturing information from different spatial regions and on different scales. Our last step is to concatenate these 21 vectors into one vector $\mathbf{z} \in \mathbb{R}^{21 * K}$. The vector $\mathbf{z}$ is the ScSPM representation of the original image $\mathbf{X}$.

\subsection{BAG OF WORDS (BOW)}

Bag of (visual) words (BOW) is a feature extraction approach inspired by the bag of words representation used in text classification tasks [20]. In text applications, BOW treats a document as a collection of words independent of each other, ignoring the order and context in which the words are used. In image applications, BOW represents an image as a histograms of its local features, using feature descriptors such as SIFT. For this work we used the PHOW algorithm to extract dense SIFT feature descriptors at multiple scales.

Whereas the ScSPM algorithm represents images with respect to a dictionary, BOW represents images with respect to a vocabulary constructed from the set of training images $\chi_{T}$. Using the VLFeat software library [17], we compute PHOW feature descriptors for each image in $\chi_{T}$ and collect them in the set $\psi_{T}$. We then fix the parameter $K$ and cluster the descriptors into $K$ clusters using the $k$-means algorithm. This gives us a matrix $\mathbf{V}=\left[\mathbf{v}_{i}\right]_{i=1}^{K} \in \mathbb{R}^{n \times K}$ (where $n$ is the length of each descriptor, in our case 128) whose columns $\mathbf{v}_{i}$ are the cluster centers, or "words."

Given an image $\mathbf{X}$, we compute the set $\psi$ containing its PHOW descriptors. For each $\mathbf{y} \in \psi$, we find the "word" that it is closest to, a step referred to as quantization. We define a function $Q(\cdot)$ by which to associate $y$ with the index of its closest "word", that is,

$$
Q(\mathbf{y})=\underset{i=1, \cdots K}{\operatorname{argmin}}\left\|\mathbf{v}_{i}-\mathbf{y}\right\|_{2}
$$

After quantizing each descriptor, we compile a histogram $\mathbf{z}=\left[z_{i}\right]_{i=1}^{K}$ reflecting how many of each "word" are represented in $\mathbf{X}$, so for each $i=1, \cdots K$

$$
z_{i}=|\{\mathbf{y} \in \psi \mid Q(\mathbf{y})=i\}| .
$$

This histogram $\mathbf{z} \in \mathbb{R}^{K}$ is the BOW representation of the image $\mathbf{X}$.

\section{EXPERIMENTS}

We compared the effectiveness of the ScSPM and BOW algorithms for image representations by using them to classify a four-class set of ship images chipped from satellite imagery. The data set contains 200 images per class, and each image is repeated under various degrees of pre-processing to give four separate data sets; the original images have non-uniform dimensions and have ships pointed in every direction, the rotated images have all the ships pointing up, the cropped images have some excess background removed, and the resized images are all $300 \times 150$ pixels and have bow, stern, port, and starboard points on each ship aligned. This data set is described in more detail in [14].

We tested each representation algorithm with two different values of $K$ (1000 and 2000 for BOW and 1024 and 2048 for ScSPM), which represents the dictionary size for ScSPM and the vocabulary size for 
BOW. For each pre-processing type, we divided the images into $80 \% / 20 \%$ splits, using 160 images per class for training and the remainder for testing. We classified the testing images with an SVM, implemented with LibSVM [2], using a linear kernel. We classified each data set five times, each time with a different split used for testing. Table 1 shows the average classification accuracy over the five runs, for each representation algorithm, dictionary or vocabulary size, and data type.

One distinction between the ScSPM and BOW algorithms is that ScSPM preserves some spatial information, but BOW does not. It is not surprising that on the original data set there is a four percentage point drop in average accuracy from BOW to ScSPM when $K=1000$ or 1024, and a six percentage point drop when $K=2000$ or 2048 . This is because the original data is not spatially uniform, so different regions of the ships are not in the same locations from image to image. For the other data sets, all of which have some spatial uniformity, the results between the two algorithms are similar, with no clear advantage to either.

Table 1. Average classification accuracies on various data sets using either BOW or ScSPM image representations.

\begin{tabular}{|c|c|c|}
\hline \multicolumn{3}{|c|}{ BOW } \\
\hline & $\bar{~} \bar{K}=1000$ & $\bar{~} \bar{K}=2000$ \\
\hline Original Data & 76.0 & 79.4 \\
\hline Rotated & 91.5 & 91.5 \\
\hline Cropped & 92.3 & 92.8 \\
\hline Resized & 94.0 & 94.8 \\
\hline \multicolumn{3}{|c|}{ ScSPM } \\
\hline & $K=1024$ & $K=2048$ \\
\hline Original Data & 72.0 & 73.3 \\
\hline Rotated & 90.3 & 90.0 \\
\hline Cropped & 93.9 & 94.1 \\
\hline Resized & 95.0 & 94.3 \\
\hline
\end{tabular}

\section{CONCLUSION}

This report contains results from experiments testing the hypothesis that the ScSPM algorithm, which produces sparse coded image representations with learned dictionaries, will provide improvement to classification accuracy over the BOW methods tested on ship imagery in $[13,14]$. This hypothesis does not hold, and our results suggest that ScSPM is not as effective as BOW on data with no spatial alignment. On aligned data the two algorithms perform similarly; however, the performance of ScSPM algorithm may be improved by different parameter selections. Many variations of BOW were detailed and tested in [13], and the variant described in this report produced the best results of all those tested. There are several possible ways to improve ScSPM, none of which have been tried on this ship data. Future experimentation will test other ways to solve the dictionary learning Problem (4) as well as the sparse coding Problem (3). 


\section{REFERENCES}

1. Bosch, A., A. Zisserman, and X. Muoz. 2007. "Image Classification using Random Forests and Ferns." Proceedings of the IEEE 11th International Conference on Computer Vision (pp. 1-8). October 14-21, Rio de Janeiro, Brazil.

2. Chang, C. and C. Lin. 2011. "LIBSVM: A Library for Support Vector Machines," ACM Transactions on Intelligent Systems and Technology, vol. 2, no. 3, pp. 27:1-27:27. Software available at http://www.csie.ntu.edu.tw/ cjlin/libsvm. Accessed 11-12-2014.

3. Donoho, D. L. 2004. "For Most Large Underdetermined Systems of Linear Equations the Minimal $l_{1}$-norm Solution is Also the Sparsest Solution," Communications on Pure and Applied Mathematics, vol. 59, no. 6, pp. 797-829.

4. Elad, M. and M. Aharon. 2006. "Image Denoising via Sparse and Redundant Representations Over Learned Dictionaries," IEEE Transactions on Image Processing, vol. 15, no. 12, pp. 3736-3745.

5. Harguess, J. and K. Rainey. 2011. "Are Face Recognition Methods Useful for Classifying Ships?" Proceedings of the 2011 IEEE Applied Imagery Pattern Recognition Workshop: Imaging for Decision Making (pp. 1-7). October 11-13, Washington, DC.

6. Ji, Z., J. Theiler, R. Chartrand, G. Kenyon, and S. Brumby. 2013. "Decoupling Sparse Coding of SIFT Descriptors for Large-Scale Visual Recognition." Proceedings of SPIE Volume 8750, Independent Component Analyses, Compressive Sampling, Wavelets, Neural Net, Biosystems, and Nanoengineering XI. April 29, Baltimore, MD.

7. Lazebnik, S., C. Schmid, and J. Ponce. 2006. "Beyond Bags of Features: Spatial Pyramid Matching for Recognizing Natural Scene Categories." IEEE Computer Society Conference on Computer Vision and Pattern Recognition, vol. 2 (pp. 2169-2178). New York, NY.

8. Lowe, D. G. 2004. "Distinctive Image Features from Scale-Invariant Keypoints," International Journal of Computer Vision, vol. 60, no. 2, pp. 91-110.

9. Mairal, J., F. Bach, J. Ponce, and G. Sapiro. 2009. "Online Dictionary Learning for Sparse Coding." Proceedings of the 26th Annual International Conference on Machine Learning (pp. 689-696). June 14-18, Montreal, Quebec, Canada.

10. Mairal, J., F. Bach, J. Ponce, and G. Sapiro. 2010. "Online Learning for Matrix Factorization and Sparse Coding," Journal of Machine Learning Research, vol. 11, pp. 19-60.

11. Mairal, J., F. Bach, J. Ponce, G. Sapiro, and A. Zisserman. 2008. "Discriminative Learned Dictionaries for Local Image Analysis." Proceedings of the 2008 IEEE Conference on Computer Vision and Pattern Recognition (pp. 1-8). 23-28 June, Anchorage, AK.

12. Rainey, K., S. Parameswaran, and J. Harguess. 2014. "Maritime Vessel Recognition in Degraded Satellite Imagery." Proceedings of SPIE Volume 9090, Automatic Target Recognition XXIV. March 13, Baltimore, MD.

13. Rainey, K., S. Parameswaran, and J. Harguess. 2014. "Vessel Classification in Overhead Satellite Imagery,” Technical Report 2062, Space and Naval Warfare Systems Center Pacific (SSC Pacific). San Diego, CA. 
14. Rainey, K., S. Parameswaran, J. Harguess, and J. Stastny. 2012. "Vessel Classification in Overhead Satellite Imagery Using Learned Dictionaries." Proceedings of SPIE Volume 8499, Applications of Digital Image Processing XXXV. October 15, San Diego, CA.

15. Rainey, K. and J. Stastny. 2011. "Object Recognition in Ocean Imagery Using Feature Selection and Compressive Sensing." Proceedings of the 2011 IEEE Applied Imagery Pattern Recognition Workshop: Imaging for Decision Making (pp. 1-6). October 11-13, Washington, DC.

16. Schölkopf, B., A. J. Smola, R. C. Williamson, and P. L. Bartlett. 2000. "New Support Vector Algorithms," Neural Computation, vol. 12, no. 5, pp. 1207-1245.

17. Vedaldi, A. and B. Fulkerson. 2008. "VLFeat: An Open and Portable Library of Computer Vision Algorithms." http://www.vlfeat.org. Accessed 11-12-2014.

18. Wright, J., A. Yang, A. Ganesh, S. Sastry, and Y. Ma. 2009. "Robust Face Recognition via Sparse Representation," IEEE Transactions on Pattern Analysis and Machine Intelligence, vol. 31, no. 2, pp. 210-227.

19. Yang, J., Y. Jiang, A. G. Hauptmann, and C. Ngo. 2007. "Evaluating Bag-of-Visual-Words Representations in Scene Classification." Proceedings of the International Workshop on Multimedia Information Retrieval (pp. 197-206). Augsburg, Bavaria, Germany.

20. Yang, J., Y. Jiang, A. G. Hauptmann, and C. Ngo. 2007. "Evaluating Bag-of-Visual-Words Representations in Scene Classification." Proceedings of the 2007 International Workshop on Multimedia Information Retrieval (pp. 197-206). September 28-29, Augsburg, Bavaria, Germany.

21. Yang, J., K. Yu, Y. Gong, and T. Huang. 2009. "Linear Spatial Pyramid Matching Using Sparse Coding for Image Classification." IEEE Conference on Computer Vision and Pattern Recognition (pp. 1794-1801). June 20-26, Miami, FL. 


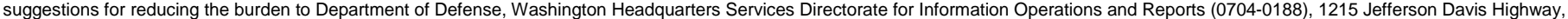

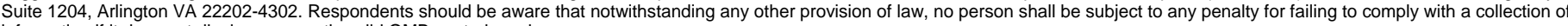

information if it does not display a currently valid OMB control number.

PLEASE DO NOT RETURN YOUR FORM TO THE ABOVE ADDRESS.

\begin{tabular}{|l|l|}
\hline 1. REPORT DATE $(D D-M M-Y Y Y Y)$ & 2. REPORT TYPE
\end{tabular}

December 2014

Final

\section{TITLE AND SUBTITLE}

\section{DATES COVERED (From - To)}

5a. CONTRACT NUMBER

Sparse Representation and Dictionary Learning as Feature Extraction in Vessel Imagery

5b. GRANT NUMBER

5c. PROGRAM ELEMENT NUMBER

\section{AUTHORS}

5d. PROJECT NUMBER

Katie Rainey

5e. TASK NUMBER

Ana Ascencio

\section{5f. WORK UNIT NUMBER}

\section{PERFORMING ORGANIZATION NAME(S) AND ADDRESS(ES)}

\section{PERFORMING ORGANIZATION} REPORT NUMBER

SSC Pacific, 53560 Hull Street, San Diego, CA 92152-5001

TR 2070

\section{SPONSORING/MONITORING AGENCY NAME(S) AND ADDRESS(ES)}

Office of Naval Research 800 North Quincy Street

Arlington, VA 22217-5000
National Geospatial-Intelligence Agency

Office of Corporate Communications

Public Affairs Branch, MS N73-OCCAE
10. SPONSOR/MONITOR'S ACRONYM(S)

11. SPONSOR/MONITOR'S REPORT NUMBER(S)

\section{DISTRIBUTIONIAVAILABILITY STATEMENT}

Approved for public release

\section{SUPPLEMENTARY NOTES}

This is a work of the United States Government and therefore is not copyrighted. This work may be copied and disseminated without restriction.

\section{ABSTRACT}

This report describes experiments designed to evaluate the usefulness of a specific algorithm for classifying images of commercial ships by class. This algorithm uses a technique known as sparse coding to represent images for classification. The sparse coding algorithm is compared with another algorithm evaluated in previous publications. The sparse coding algorithm is shown to perform approximately as well as the algorithm it is compared with and does not appear to offer any improvement. Additional research is required to identify algorithms best suited for the ship classification task.

\section{SUBJECT TERMS}

Mission Area: Situational Awareness

sparse coded features spatial pyramid spatial pyramid matching dictionary learning learned dictionaries bag of words

\begin{tabular}{|c|c|c|c|c|c|}
\hline \multicolumn{3}{|c|}{ 16. SECURITY CLASSIFICATION OF: } & \multirow{3}{*}{$\begin{array}{l}\text { 17. LIMITATION OF } \\
\text { ABSTRACT } \\
\text { U }\end{array}$} & \multirow{3}{*}{$\begin{array}{l}\text { 18. NUMBER } \\
\text { OF } \\
\text { PAGES } \\
39\end{array}$} & \multirow{2}{*}{$\begin{array}{l}\text { 19a. NAME OF RESPONSIBLE PERSON } \\
\text { Kate Rainey }\end{array}$} \\
\hline a. REPORT & b. ABSTRACT & c. THIS PAGE & & & \\
\hline $\mathrm{U}$ & $\mathrm{U}$ & $\mathrm{U}$ & & & $\begin{array}{l}\text { 19B. TELEPHONE NUMBER (Include area code) } \\
\text { (619) 553-3472 }\end{array}$ \\
\hline
\end{tabular}




\section{INITIAL DISTRIBUTION}

$84300 \quad$ Library

$85300 \quad$ Archive/Stock

55280

K. Rainey

(1)

56220 A. Ascencio

(1)

Defense Technical Information Center

Fort Belvoir, VA 22060-6218 
Approved for public release.

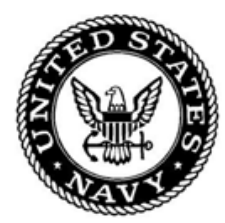

SSC Pacific San Diego, CA 92152-5001 